\title{
Upper estimates for inhomogeneous random walks confined to the positive orthant
}

\author{
Rim Essif* $\quad$ Sami Mustapha ${ }^{\dagger}$
}

\begin{abstract}
We obtain upper Gaussian estimates of transition probabilities of a spatially nonhomogeneous random walk confined to a multidimensional orthant. For the proof, we use comparison arguments based on discrete potential theory and variants of the Harnack principle.
\end{abstract}

Keywords: random walk in the positive orthant; discrete harmonic functions; discrete potential theory.

MSC2020 subject classifications: Primary 60G50; 31C35, Secondary 60G40; $30 \mathrm{~F} 10$.

Submitted to ECP on December 24, 2020, final version accepted on July 3, 2021.

\section{Introduction}

Random walks conditioned to stay in cones is an important topic in probability theory, as they appear naturally in many contexts: non intersecting paths and multidimensional random walks in Weyl chambers [33, 14, 19, 11], eigenvalues of random matrices [12], queueing theory [8], modeling of biological and physical phenomena [3, 16, 24], finance [9], etc. They have also important connections with many discrete structures (trees, permutations, maps, partitions, domino tiling, Young diagrams) which justifies the great interest they have in combinatorics; see $[4,6]$ and the references therein.

In the recent years, the case of homogeneous random walks confined to the $d$ dimensional orthant $\mathcal{O}_{+}^{d}=\left(\mathbb{Z}_{+}\right)^{d}=\left\{\left(x_{1}, \ldots, x_{d}\right) \in \mathbb{Z}^{d} ; x_{1} \geq 0, \ldots, x_{d} \geq 0\right\}$ has been subject of several important works especially for the cases $d=2,3$; see $[4,6,11,15,23$, $32,35]$.

Regarding spatially non-homogeneous walks, only few results are available; see [31] for half-space non-homogeneous walks and $[2,7,10,24]$ for quadrant walks. By spatially non-homogeneous walk on $\mathbb{Z}^{d}$, we mean a discrete-time-homogenous Markov chain on $\mathbb{Z}^{d}$ which is spatially non-homogeneous, see [29]. Let us notice that relaxing the spatial homogeneity hypothesis induces a crucial change in the recurrence/transience behavior of zero-mean random walks compared to the homogeneous case, since it is possible to build two-dimensional, zero-drift random walks with bound increments that are transient, as soon as spatial homogeneity is no longer required [17]. It is also more realistic for applications and modeling.

\footnotetext{
${ }^{*}$ Centre de Recherche en Économie et Statistique, École Nationale de la Statistique et de l'Administration Economique, FRANCE. E-mail: rim.essifi@ensae.fr

${ }^{\dagger}$ Institut de Mathématiques de Jussieu-Paris Rive Gauche, Sorbonne Université, France. E-mail: sam@math. jussieu.fr
} 
The main purpose of this paper is to establish upper Gaussian estimates for the transition kernels of a large class of spatially non-homogeneous random walks killed on the boundary of the orthant $\mathcal{O}_{+}^{d}$. We refine the approach used in the case of the half-space $\mathbb{Z}_{+}^{d}$ [31] and in the case of models with one dimensional space inhomogeneity on the positive quadrant [2].

The paper is organized as follows. Section 2 collects the potential theoretic tools used in the proof of our main result. Essentially, we introduce the notion of caloric function, the discrete parabolic Harnack principle and the discrete boundary parabolic Harnack inequality. We also state at the end of this section our main result (Theorem 2.12). In Section 3, we explicit the proof of Theorem 2.12.

Convention. Throughout this paper the letters $C, c$, possibly with suffixes, will indicate positive constants that only depend on the important parameters and may differ from place to another even in the same formula. Most often $C$ is reserved to denote large constants and $c$ small ones.

\section{Known results and statement of the main theorem}

Fix $d \in \mathbb{N}^{*}$. Let us denote by $\mathcal{O}_{+}^{d}=\left(\mathbb{Z}_{+}\right)^{d}=\left\{\left(x_{1}, \ldots, x_{d}\right) \in \mathbb{Z}^{d} ; x_{1} \geq 0, \ldots, x_{d} \geq 0\right\}$ the upper orthant in $\mathbb{Z}^{d}$. Next, consider a steps set $\Gamma \subset \mathbb{Z}^{d}$ that we assume finite, symmetric (i.e., $e \in \Gamma$ implies $-e \in \Gamma$ ) and containing 0 and all $e \in \mathbb{Z}^{d}$ such that $|e|=1$, where $|\cdot|$ is the Euclidean norm on $\mathbb{R}^{d}$. Let $\pi: \mathbb{Z}^{d} \times \mathbb{Z}^{d} \rightarrow[0,1]$ denote a transition kernel satisfying the following hypotheses:

$\mathbf{H}_{\mathbf{1}}: \pi(x, e)=0$ for all $x \in \mathbb{Z}^{d}, e \notin \Gamma ;$ and $\sum_{e \in \Gamma} \pi(x, e)=1$ for all $x \in \mathbb{Z}^{d}$,

$\mathbf{H}_{\mathbf{2}}: \pi(x,-e)=\pi(x, e)$ for all $x, e \in \mathbb{Z}^{d},(i . e ., \pi$ is symmetric $)$,

and

$\mathbf{H}_{3}$ : (ellipticity) there exists $\alpha>0$ such that $\pi(x, e) \geq \alpha$, for all $x \in \mathbb{Z}^{d}$ and $e \in \Gamma$.

Let $\left(S_{n}\right)_{n \in \mathbb{N}}$ be the Markov chain on $\mathbb{Z}^{d}$, defined by

$$
\mathbb{P}\left[S_{n+1}=x+e \mid S_{n}=x\right]=\pi(x, e), \quad x, e \in \mathbb{Z}^{d}, n=0,1, \ldots
$$

Introduce $\tau=\inf \left\{n=0,1, \cdots: S_{n} \notin \mathcal{O}_{+}^{d}\right\}$ the first exit time of the walk $\left(S_{n}\right)_{n \in \mathbb{N}}$ from $\mathcal{O}_{+}^{d}$ and then define $p_{n}(x, y):=\mathbb{P}_{x}\left[S_{n}=y ; \tau>n\right], n=1,2, \ldots, x, y \in \mathcal{O}_{+}^{d}$, the transition kernel of the walk $\left(S_{n}\right)_{n \in \mathbb{N}}$ killed outside $\mathcal{O}_{+}^{d}$. Our principal aim in this work is to give upper Gaussian estimates of the kernel $p_{n}(x, y)$. Our approach is based essentially on discrete potential theory. We first define the main potential theoretic relevant objects and recall the most important ingredients that we shall use in our estimates within the framework set by our three assumptions. We then state our main theorem. We shall also elaborate on the relevance of $\mathbf{H}_{1}, \mathbf{H}_{2}, \mathbf{H}_{3}$.

\subsection{Discrete potential Theory}

Let $\Gamma \subset \mathbb{Z}^{d}$ be a finite steps set as above and $\pi: \mathbb{Z}^{d} \times \mathbb{Z}^{d} \rightarrow[0,1]$ a transition kernel such that hypotheses $\mathbf{H}_{1}, \mathbf{H}_{2}$ and $\mathbf{H}_{3}$ hold. Denote by $L$ the generator corresponding to the Markov chain defined by (2.1), which is the difference operator acting on functions $f: \mathbb{Z}^{d} \rightarrow \mathbb{R}$ as

$$
L f(x)=\sum_{e \in \Gamma} \pi(x, e)(f(x+e)-f(x)), \quad x \in \mathbb{Z}^{d} .
$$

The existence of a global positive adjoint solution associated to the difference operator (2.2) plays a key role in our analysis. According to [30], one has the following fact.

Fact 2.1. [30, §3.2] There exists a positive function $(m(x))_{x \in \mathbb{Z}^{d}}$ which satisfies the adjoint 
equation

$$
\sum_{e \in \Gamma} \pi(x-e, e) m(x-e)=m(x), \quad x \in \mathbb{Z}^{d} .
$$

This function is unique up to a multiplicative constant.

To the function $m$, one can associate a volume function:

$$
v(x, r)=\sum_{y \in B_{r}(x)} m(y), x \in \mathbb{Z}^{d}, r>0,
$$

where $B_{r}(x):=\left\{y \in \mathbb{Z}^{d},|y-x|<r\right\}$ denotes the Euclidean ball in $\mathbb{Z}^{d}$ centered on $x$ and of radius $r>0$. The function $v$ satisfies the volume doubling property [30, Theorem 8]

$$
v(x, 2 r) \leq C v(x, r), \quad x \in \mathbb{Z}^{d} ; r>0 .
$$

Remark 2.2. One can check that inequality (2.4) implies a polynomial growth of the volume function, more precisely, there exists a constant $C>0$ and an integer $N \in \mathbb{N}^{*}$ such that for all $x \in \mathbb{Z}^{d}$ and $R \geq r>0$, one has

$$
v(x, R) \leq C\left(\frac{R}{r}\right)^{N} v(x, r) .
$$

As a consequence of inequality (2.5), one gets the following volume comparison inequality

$$
v(y, r) \leq C\left(1+\frac{|x-y|}{r}\right)^{N} v(x, r), x, y \in \mathbb{Z}^{d} ; r>0 .
$$

Denote by $p_{n}^{\mathbb{Z}^{d}}(x, y):=\mathbb{P}_{x}\left[S_{n}=y\right]$ the global transition kernel. The positive adjoint solution $m$ and the volume function $v$ allow to establish an upper Gaussian estimate of $p_{n}^{\mathbb{Z}^{d}}(x, y)$ of the form [30, §3.2]:

Theorem 2.3 (Estimate of the global transition kernel). There exists a positive constant $C=C(d, \alpha, \Gamma)$ such that for all $n \in \mathbb{N}$ and $x, y \in \mathbb{Z}$, one has

$$
p_{n}^{\mathbb{Z}^{d}}(x, y) \leq \frac{C m(y)}{v(x, \sqrt{n})} \exp \left(-\frac{|x-y|^{2}}{C n}\right), \quad x, y \in \mathbb{Z}^{d} ; n \geq 1 .
$$

Let us now introduce the different notions of boundary that we are going to use. Note that the definitions that we employ do not coincide exactly with those used in combinatorics, for example, according to our definition, in the case of the simple walk, the vertex $(-1,-1, \cdots,-1)$ is not part of the boundary of $\mathcal{O}_{+}^{d}$. More precisely:

- Two points $x, y \in \mathbb{Z}^{d}$ are said to be adjacent if the distance between them is unity. A subset $A \subset \mathbb{Z}^{d}$ will be called connected if for any two points of $A$ there is a path consisting of segments of unit length connecting them in such a manner that the end-points of these segments are all in $A$. A set of points is a domain if it is connected.

- We define the boundary $\partial A$ of a domain $A$ by

$$
\partial A=\left\{x \in A^{c}, x=a+e, \text { for some } a \in A \text { and } e \in \Gamma\right\} .
$$

The closure of $A$ will be denoted by $\bar{A}$ and defined by $\bar{A}=A \cup \partial A$.

- For a subset $B=A \times \rrbracket a, b \rrbracket \subset \mathbb{Z}^{d} \times \mathbb{Z}$, where $A$ denotes a domain in $\mathbb{Z}^{d}$ and $a<b \in \mathbb{Z}$, we define the lateral boundary $\partial_{l} B$ and the parabolic boundary $\partial_{p} B$ of $B$ by

$$
\partial_{l} B=\partial A \times \rrbracket a, b \llbracket, \quad \partial_{p} B=\partial_{l} B \cup(\bar{A} \times\{a\}),
$$

and we set $\bar{B}=B \cup \partial_{p} B$. 
Inhomogeneous random walks on the orthant

More generally, we can define the parabolic boundary of a space-time domain $D \subset \mathbb{Z}^{d} \times \mathbb{Z}$ by $\partial_{p} D=\left\{(x, t) \in D^{c}, \quad(x+e, t+1) \in D\right.$, for some $\left.e \in \Gamma\right\}$. This definition is the natural extension of the notion of parabolic boundary of a domain $D \subset \mathbb{R}^{d} \times \mathbb{R}$ used in the theory of second order parabolic equations [13], which is defined as the set of all points $(x, t) \in \partial D$ such that there is a continuous curve lying in $D \cup\{(x, t)\}$ with initial point at $(x, t)$ along which $t$ is non-decreasing. This explains why $\bar{A} \times\{b\}$ is not included in $\partial_{p} B$ and does not appear in (2.8).

The concepts of discrete harmonic and caloric functions naturally associated with the random walk $\left(S_{n}\right)_{n \in \mathbb{N}}$ are defined as follows, see [30, 31].

Definition 2.4. Let $L$ be a difference operator as in (2.2), let $A \subset \mathbb{Z}^{d}$ denote a domain in $\mathbb{Z}^{d}$ and $f$ a real valued function defined on $\bar{A}$. We say that $f$ is $L$-harmonic on $A$ if

$$
L f(x)=0, \quad x \in A \text {. }
$$

Consider $B=A \times \rrbracket a, b \rrbracket \subset \mathbb{Z}^{d} \times \mathbb{Z}$ and $F: \bar{B} \rightarrow \mathbb{R}$. We say that $F$ is $L$-caloric in $B$ if

$$
L(F(., k))(x)-(F(x, k+1)-F(x, k))=0, \quad(x, k) \in A \times \rrbracket a, b \llbracket .
$$

The following theorem (see [21, Theorem 4.2], [22, Theorem 4.4]), which is a key tool in proving our theorem, is a random walk version of the well-known and fundamental property of nonnegative solutions of second order parabolic equations in non-divergence form [20].

Theorem 2.5 (Parabolic Harnack principle). Let $F$ be a nonnegative $L$-caloric function on $B_{2 r}(y) \times \rrbracket s-4 r^{2}, s \rrbracket,(y, s) \in \mathbb{Z}^{d} \times \mathbb{Z}, r \geq 1$. Then

$\sup \left\{F(x, k) ;(x, k) \in B_{r}(y) \times \rrbracket s-3 r^{2}, s-2 r^{2} \llbracket\right\} \leq C \inf \left\{F(x, k) ;(x, k) \in B_{r}(y) \times \rrbracket s-r^{2}, s \llbracket\right\}$,

where $C=C(d, \alpha, \Gamma)>0$.

To get an idea of the probabilistic meaning of estimate (2.9), let us associate to the process $\left(S_{n}\right)_{n \in \mathbb{N}}$ the corresponding space-time process $\{\hat{S}(n), n \in \mathbb{N}\}=\left(S_{n}, n_{0}-n\right)_{n \in \mathbb{N}}$ starting at $\left(x_{0}, n_{0}\right) \in \mathbb{Z}^{d} \times \mathbb{Z}$ and consider $\hat{\xi}=\inf \left\{j \geq 0: \hat{S}(j) \in \partial_{p}\left(B_{2 r}(y) \times \rrbracket s-4 r^{2}, s \rrbracket\right)\right\}$. Then the parabolic Harnack inequality holds if the following holds: for all $\left(x_{1}, k_{1}\right) \in$ $B_{r}(y) \times \rrbracket s-3 r^{2}, s-2 r^{2} \llbracket,\left(x_{2}, k_{2}\right) \in B_{r}(y) \times \rrbracket s-r^{2}, s \llbracket,(z, n) \in \partial_{p}\left(B_{2 r}(y) \times \rrbracket s-4 r^{2}, s \rrbracket\right)$ $\left(n<k_{1}\right)$, the probability $\mathbb{P}_{x_{1}, k_{1}}[\hat{S}(\hat{\xi})=(z, n)]$ is dominated by $C \mathbb{P}_{x_{2}, k_{2}}[\hat{S}(\hat{\xi})=(z, n)]$.

The second ingredient we need in the proof of our main theorem is the following boundary Harnack inequality.

Theorem 2.6 (Boundary Harnack principle). Let $(y, s) \in \partial \mathcal{O}_{+}^{d} \times \mathbb{Z}, r \geq 1$. Suppose $K>0$ large enough. Assume that $F_{1}, F_{2}$ are two nonnegative $L$-caloric functions in

$$
\left(B_{3 K r}(y) \cap \mathcal{O}_{+}^{d}\right) \times \rrbracket s-9 K^{2} r^{2}, s+9 K^{2} r^{2} \rrbracket
$$

and that $F_{1}$ is identically equal to zero on $\left(B_{2 K r}(y) \cap \partial \mathcal{O}_{+}^{d}\right) \times \llbracket s-4 K^{2} r^{2}, s+4 K^{2} r^{2} \rrbracket$. Then

$$
\sup \left\{\frac{F_{1}(x, k)}{F_{2}(x, k)}, \quad(x, k) \in\left(B_{r}(y) \cap \mathcal{O}_{+}^{d}\right) \times \llbracket s-r^{2}, s \rrbracket\right\} \leq C \frac{F_{1}\left(y_{K r}, s+2 K^{2}\lfloor r\rfloor^{2}\right)}{F_{2}\left(y_{K r}, s-2 K^{2}\lfloor r\rfloor^{2}\right)},
$$

where $C=C(d, \alpha, \Gamma)>0$. Here $y_{r}=y+\lfloor r\rfloor(1, \ldots, 1)$ and $\lfloor r\rfloor$ denotes the integer part of the real $r$.

The proof of Estimate (2.10) is a straightforward adaptation of the proof of the corresponding estimate for non-negative $L$-caloric functions in cylindrical domains given 
in $[30, \S 5]$. A special case that plays a crucial role in the proof of the general case is the case where $F_{2}=1$. In this case, relation (2.10) is referred to as a Carleson inequality. The proof of Carleson inequality relies on the following lower bound of the hitting probabilities of the process $\{\hat{S}(n), n \in \mathbb{N}\}$. Let $\hat{\zeta}$ denote the first exit time of $\{\hat{S}(n), n \in \mathbb{N}\}$ from $\left(\mathcal{O}_{+}^{d} \cap B_{2 r}(y)\right) \times \llbracket s-4 r^{2} ; s+4 r^{2} \rrbracket$, where $(y, s) \in \partial \mathcal{O}_{+}^{d} \times \mathbb{Z}$ and $r \geq 1$. Then

$$
\mathbb{P}_{x, n}\left[\hat{S}(\hat{\zeta}) \in\left(\partial \mathcal{O}_{+}^{d} \cap B_{2 r}(y)\right) \times \llbracket s-4 r^{2} ; s+4 r^{2} \rrbracket\right] \geq \theta
$$

for all $(x, n) \in\left(\mathcal{O}_{+}^{d} \cap B_{r}(y)\right) \times \llbracket s-r^{2} ; s+r^{2} \rrbracket$, where $\theta=\theta(d, \alpha, \Gamma)>0$. It follows from (2.11) that if $F$ is nonnegative caloric function in $\left(\mathcal{O}_{+}^{d} \cap B_{3 r}(y)\right) \times \llbracket s-9 r^{2} ; s+9 r^{2} \rrbracket$ vanishing on the portion of the parabolic boundary $\left(\partial \mathcal{O}_{+}^{d} \cap B_{2 r}(y)\right) \times \llbracket s-4 r^{2} ; s+4 r^{2} \rrbracket$, then the maximum of $F$ on $\left(\mathcal{O}_{+}^{d} \cap B_{r}(y)\right) \times \llbracket s-r^{2} ; s+r^{2} \rrbracket$ is dominated by its maximum on $\left(\mathcal{O}_{+}^{d} \cap B_{2 r}(y)\right) \times \llbracket s-4 r^{2} ; s+4 r^{2} \rrbracket$ multiplied by a factor $\rho=\rho(d, \alpha, \Gamma)<1$. An argument from Fabes, Safonov and Yuan [13] allows then to deduce the Carleson inequality.

The proof of (2.10) in all generality results from a combination of the Harnack inequality, the Carleson inequality and a refinement of (2.11) concerning the hitting probabilities of the process $\{\hat{S}(n), n \in \mathbb{N}\}$ which can be stated as follows. For $(y, s) \in$ $\partial \mathcal{O}_{+}^{d} \times \mathbb{Z}, r$ and $K \geq 1$, let $D_{K r, r}$ denote the set of points of $\left(\mathcal{O}_{+}^{d} \cap B_{K r}(y)\right) \times \llbracket s-K^{2} r^{2} ; s \rrbracket$ satisfying $\operatorname{dist}\left(x, \partial \mathcal{O}_{+}^{d}\right)<r$. Set $\Delta_{K r, r}=\left\{(x, t) \in \partial_{p} D_{K r, r}\right.$, dist $\left.\left(x, \partial \mathcal{O}_{+}^{d}\right) \geq r\right\}$ and $\Delta_{K r, r}^{\prime}=\left\{(x, t) \in \partial_{p} D_{K r, r}, 0<\operatorname{dist}\left(x, \partial \mathcal{O}_{+}^{d}\right)<r\right\}$. Let $\hat{\tau}_{D_{K r, r}}$ denote the first exit time of $(\hat{S}(n))_{n \in \mathbb{N}}$ from $D_{K r, r}$. Then, for all $(x, n) \in\left(\mathcal{O}_{+}^{d} \cap B_{r}(y)\right) \times \llbracket s-r^{2} ; s \rrbracket$

$$
\mathbb{P}_{x, n}\left[\hat{S}\left(\hat{\tau}_{D_{K r, r}}\right) \in \Delta_{K r, r}\right] \geq \mathbb{P}_{x, n}\left[\hat{S}\left(\hat{\tau}_{D_{K r, r}}\right) \in \Delta_{K r, r}^{\prime}\right],
$$

provided that $K \geq K_{0}$, where $K_{0}=K_{0}(d, \alpha, \Gamma)$. Note that the constant $C$ which appears in (2.10) does not depend on $K$ (see [30, §5.3]).

A significant difference between the boundary Harnack inequality (2.10) and the Harnack inequality (2.9) is that (2.10) involves two reference points. However, if the second function $F_{2}$ is independent of time, i.e., $F_{2}$ is harmonic (this will be the case in our applications), this difference no longer exists and (2.10) has a more natural form that takes into account only the time-lag that characterizes the parabolic Harnack inequalities. The boundary principle can then be viewed as a Harnack inequality for $\left(S_{n}\right)_{n \in \mathbb{N}}$ conditioned to stay in the orthant via Doob $h$-transform. Such a choice for $F_{2}$ is guaranteed by the following result which ensures the existence and uniqueness of a positive harmonic function in $\mathcal{O}_{+}^{d}$ vanishing on the boundary $\partial \mathcal{O}_{+}^{d}$ (see [5, Theorem 1.1]).

Theorem 2.7. There exists a positive $L$-harmonic function $u$ defined in $\mathcal{O}_{+}^{d}$ and vanishing on $\partial \mathcal{O}_{+}^{d}$. This function is unique up to a multiplicative constant.

It should however be emphasized that apart from the case where the random walk is reversible, it is not possible to establish the Gaussian estimates thanks to $h$-transform techniques by following the methods of $[18,34]$ which are based on the use of weighted Dirichlet forms. To get around this difficulty which is specific to inhomogeneous walks, we need to consider the process which is dual to $\left(S_{n}\right)_{n \in \mathbb{N}}$ with respect to $m$ and rely on the potential theory attached to it.

\subsection{Adjoint potential theory}

The existence of the global positive adjoint solution $m$ (Fact 2.1) allows us to define a dual process $\left(\tilde{S}_{n}\right)_{n \in \mathbb{N}}$ by setting

$$
\mathbb{P}\left[\tilde{S}_{n+1}=x+e \mid \tilde{S}_{n}=x\right]=\tilde{\pi}(x, e), \quad x, e \in \mathbb{Z}^{d}, \quad n \in \mathbb{N},
$$


where

$$
\tilde{\pi}(x, e)=\left\{\begin{array}{cc}
\pi(x+e, e) \frac{m(x+e)}{m(x)}, & e \in \Gamma, \\
0 & \text { otherwise. }
\end{array}\right.
$$

One can easily check that Equation (2.3) implies that $\tilde{\pi}$ satisfies $\mathbf{H}_{\mathbf{1}}$ and the ellipticity condition $\mathbf{H}_{\mathbf{3}}$ (with $\alpha^{2}$ instead of $\alpha$ ). Note that $\mathbf{H}_{\mathbf{2}}$ is not satisfied by the chain $\left(\tilde{S}_{n}\right)_{n \in \mathbb{N}}$. Introduce now $\tilde{\tau}=\inf \left\{n=0,1, \cdots: \tilde{S}_{n} \notin \mathcal{O}_{+}^{d}\right\}$ the first exit time of the walk $\left(\tilde{S}_{n}\right)_{n \in \mathbb{N}}$ from $\mathcal{O}_{+}^{d}$. The transition probabilities of the walk $\tilde{S}_{n}$ killed outside $\mathcal{O}_{+}^{d}$ satisfy

$$
\tilde{p}_{n}(x, y):=\mathbb{P}_{x}\left[\tilde{S}_{n}=y ; \tilde{\tau}>n\right]=\frac{p_{n}(y, x) m(y)}{m(x)}, \quad n=1,2, \cdots, x, y \in \mathcal{O}_{+}^{d} .
$$

Note that the operator

$$
\widetilde{L} f(x)=\sum_{e \in \Gamma} \tilde{\pi}(x, e)(f(x+e)-f(x)), \quad f: \mathbb{Z}^{d} \mapsto \mathbb{R},
$$

is the adjoint operator of $L$ in the space $L^{2}\left(\mathbb{Z}^{d}, m\right)$. Similarly to definitions 2.4 , we introduce in the following the concepts of harmonic and caloric functions associated with the dual process $\left(\tilde{S}_{n}\right)_{n \in \mathbb{N}}$. Except in the reversible case, they are not related to $L$-harmonic and caloric functions.

Definition 2.8. Let $A \subset \mathbb{Z}^{d}$ denote a domain in $\mathbb{Z}^{d}$ and $\tilde{f}: \bar{A} \rightarrow \mathbb{R}$. We say that $\tilde{f}$ is $\tilde{L}$-harmonic on $A$ if

$$
\tilde{L} \tilde{f}(x)=0, x \in A .
$$

Consider $B=A \times \rrbracket a, b \rrbracket \subset \mathbb{Z}^{d} \times \mathbb{Z}$ and $\tilde{F}: \bar{B} \rightarrow \mathbb{R}$. We say that $\tilde{F}$ is $\tilde{L}$-caloric in $B$ if

$$
\tilde{L}(\tilde{F}(., k))(x)-(\tilde{F}(x, k+1)-\tilde{F}(x, k))=0, \quad(x, k) \in A \times \rrbracket a, b \llbracket .
$$

As a consequence of the parabolic Harnack principle (Theorem 2.5), the boundary Harnack principle (Theorem 2.6) and the fact that the kernel $\tilde{\pi}$ satisfies the ellipticity condition $\mathbf{H}_{3}$, one obtains the parabolic Harnack principle for $\tilde{L}$-caloric functions, see [30]. Note that the proof given in [30] is based on a representation formula which holds for all normalized parabolic adjoint solutions of $L$ (see [30, Formula (3.2)]) and it is easy to see that every $\tilde{L}$-caloric function defines a normalized parabolic adjoint solution of $L$ in the sense of [30]. It is this representation formula which makes it possible to demonstrate the Harnack inequality for nonnegative $\tilde{L}$-caloric functions without using any symmetry hypothesis.

Theorem 2.9 (Adjoint parabolic Harnack principle). Let $\tilde{F}$ be a nonnegative $\tilde{L}$-caloric function in $B_{2 r}(y) \times \rrbracket s-4 r^{2}, s \rrbracket$, where $(y, s) \in \mathbb{Z}^{d} \times \mathbb{Z}$ and $r \geq 1$. Then, there exists a positive constant $C=C(d, \alpha, \Gamma)$ such that

$\sup \left\{\tilde{F}(x, k) ;(x, k) \in B_{2 r}(y) \times \rrbracket s-3 r^{2}, s-2 r^{2} \llbracket\right\} \leq C \inf \left\{\tilde{F}(x, k) ;(x, k) \in B_{r}(y) \times \rrbracket s-r^{2}, s \llbracket\right\}$.

On the other hand, the proof of Theorem 4 of [30] is based only on the parabolic Harnack inequality and the maximum principle. It is easy to see that $\tilde{L}$-caloric functions satisfy this last principle. The proof given in [30] therefore applies when we deal with $\tilde{L}$-caloric functions. We successively establish the analogues of (2.11) and (2.12) for the dual process $\left(\tilde{S}_{n}\right)_{n \in \mathbb{N}}$ and we obtain the following adjoint boundary Harnack principle.

Theorem 2.10 (Adjoint boundary Harnack principle). Let $(y, s) \in \partial \mathcal{O}_{+}^{d} \times \mathbb{Z}, r \geq 1$ and $K>0$ large enough. Assume that $\tilde{F}_{1}, \tilde{F}_{2}$ are two nonnegative $\tilde{L}$-caloric functions in

$$
\left(\mathcal{O}_{+}^{d} \cap B_{3 K r}(y)\right) \times \rrbracket s-9 K^{2} r^{2}, s+9 K^{2} r^{2} \rrbracket,
$$


and that $\tilde{F}_{1}$ is identically equal to zero on $\left(\partial \mathcal{O}_{+}^{d} \cap B_{2 K r}(y)\right) \times \llbracket s-4 K^{2} r^{2}, s+4 K^{2} r^{2} \rrbracket$. Then, there exists a positive constant $C=C(d, \alpha, \Gamma)$ such that

$$
\sup \left\{\frac{\tilde{F}_{1}(x, k)}{\tilde{F}_{2}(x, k)}, \quad(x, k) \in\left(B_{r}(y) \cap \mathcal{O}_{+}^{d}\right) \times \llbracket s-r^{2}, s \rrbracket\right\} \leq C \frac{\tilde{F}_{1}\left(y_{K r}, s+2 K^{2}\lfloor r\rfloor^{2}\right)}{\tilde{F}_{2}\left(y_{K r}, s-2 K^{2}\lfloor r\rfloor^{2}\right)} .
$$

The following result is an analogue of Theorem 9 in [31]. The proof given in the case of the half space $\mathbb{Z}_{+}^{d}$ immediately generalizes to our setting.

Theorem 2.11. There exists a positive $\tilde{L}$-harmonic function $\tilde{u}$ defined in $\mathcal{O}_{+}^{d}$ and vanishing on $\partial \mathcal{O}_{+}^{d}$. This solution is unique up to a multiplicative constant.

\subsection{The main theorem}

We can now state our main theorem.

Theorem 2.12. Let $\Gamma \subset \mathbb{Z}^{d}$ be a finite symmetric steps set containing 0 and all unit vectors $e \in \mathbb{Z}^{d}$ and let $\pi: \mathbb{Z}^{d} \times \mathbb{Z}^{d} \rightarrow[0,1]$ be a transition kernel such that hypotheses $\mathbf{H}_{1}, \mathbf{H}_{2}$ and $\mathbf{H}_{3}$ hold. Denote by $\left(S_{n}\right)_{n \in \mathbb{N}}$ the Markov chain on $\mathbb{Z}^{d}$, defined by (2.1) and consider $\tau$ the first exit time of $\left(S_{n}\right)_{n \in \mathbb{N}}$ from $\mathcal{O}_{+}^{d}$. Then there exists a positive constant $C$ depending only on $d, \alpha, \Gamma$ such that for all $x, y \in \mathcal{O}_{+}^{d}$ and $n \in \mathbb{N}^{*}$,

$$
p_{n}(x, y):=\mathbb{P}_{x}\left[S_{n}=y ; \tau>n\right] \leq \frac{C u(x) \widetilde{u}(y) m(y)}{u\left(x_{\sqrt{n}}\right) \widetilde{u}\left(y_{\sqrt{n}}\right) v(x, \sqrt{n})} \exp \left(-\frac{|x-y|^{2}}{C n}\right),
$$

where $x_{\sqrt{n}}=x+\lfloor\sqrt{n}\rfloor(1, \ldots, 1), y_{\sqrt{n}}=y+\lfloor\sqrt{n}\rfloor(1, \ldots, 1)$ and the functions $m, v, u$ and $\tilde{u}$, are the ones defined in $\$ 2.1,2.2$.

Remark 2.13. (i) It is easy to see that in the case of homogeneous random walks, the function $m$ is identically equal to 1 and that the functions $u$ and $\tilde{u}$ are identical. It follows that the volume induced by $m$ is the standard one, i.e., $v(x, \sqrt{n}) \simeq n^{d / 2}$. On the other hand, it follows from [35] and [11] that after a linear change of coordinates reducing the covariance matrix of $\left(S_{n}\right)_{n \in \mathbb{N}}$ to the identity, the asymptotic behavior of the function $u$ is the same as that of $u_{\mathcal{C}}$, the "réduite" of a convex conical region $\mathcal{C} \subset \mathbb{R}^{d}$. This réduite is given by $u_{\mathcal{C}}(x)=|x|^{p} m(x /|x|)$, where $p>1$ and $m: \mathcal{C} \cap \mathbb{S}^{d-1} \rightarrow \mathbb{R}$ is a $C^{2}$-function, positive in the interior of $\mathcal{C} \cap \mathbb{S}^{d-1}$ and vanishing on $\partial \mathcal{C} \cap \mathbb{S}^{d-1}$. It is easy to see (because $\mathcal{C}$ is convex) that $u_{\mathcal{C}}(x)>c \operatorname{dist}(x, \partial \mathcal{C})^{p}$ (see [11, Lemma 19]) which implies that the factor $u\left(x_{\sqrt{n}}\right) \widetilde{u}\left(y_{\sqrt{n}}\right) \geq c n^{p}$ and the transition kernel $p_{n}(x, y)$ can be dominated as follows

$$
p_{n}(x, y) \leq \frac{C u_{\mathcal{C}}(x) u_{\mathcal{C}}(y)}{n^{p+d / 2}} \exp \left(-\frac{|x-y|^{2}}{C n}\right)
$$

in the homogeneous case, see [35].

(ii) In the case of a spatially inhomogeneous random walk in the upper half-space $\mathbb{Z}_{+}^{d}=\left\{\left(x_{1}, \ldots, x_{d}\right) \in \mathbb{Z}^{d} ; x_{d} \geq 0\right\}$, the function $u$ is given by $u(x)=x_{d}+1$. Denote by $\widetilde{u}^{d}$ the analogue of $\widetilde{u}$ for the upper half-space and by $p_{n}^{\mathbb{Z}_{+}^{d}}(x, y):=\mathbb{P}_{x}\left[S_{n}=y ; \tau_{\mathbb{Z}_{+}^{d}}>n\right], \tau_{\mathbb{Z}_{+}^{d}}$ being the first exit time of $\left(S_{n}\right)_{n \in \mathbb{N}}$ from $\mathbb{Z}_{+}^{d}$. The refinements of [31] that we introduce here allow to obtain the following improvement of the upper Gaussian estimates obtained in [31],

$$
p_{n}^{\mathbb{Z}_{+}^{d}}(x, y) \leq \frac{C\left(x_{d}+1\right) \widetilde{u}^{\mathbb{Z}_{+}^{d}}(y) m(y)}{\left(x_{d}+\sqrt{n}\right) \widetilde{u}^{\mathbb{Z}_{+}^{d}}\left(y_{\sqrt{n}}\right) v(x, \sqrt{n})} \exp \left(-\frac{|x-y|^{2}}{C n}\right) .
$$

(iii) In the article [30] in addition to the upper estimate (2.7), a lower Gaussian estimate was also obtained for the global kernel $p_{n}^{\mathbb{Z}^{d}}(x, y)$. The first step in establishing this lower 
Inhomogeneous random walks on the orthant

estimate is to get a lower bound for the transition kernel on the diagonal $\{x=y\}$ by integrating the upper estimate (2.7) outside a ball of radius $\approx \sqrt{n}$. Unfortunately, even in the case of the half-space $\mathbb{Z}_{+}^{d}$, it is not easy to exploit the upper estimate in this way. The reason is simply that we no longer have at our disposal the doubling properties for the two new factors that appear (i.e. $u\left(x_{\sqrt{n}}\right), \widetilde{u}\left(y_{\sqrt{n}}\right)$. However we conjecture that a refinement of our approach could lead to a lower estimate of the same type.

\subsection{Discussion}

In the following we discuss the relevance of assumptions $\mathbf{H}_{1}, \mathbf{H}_{2}$ and $\mathbf{H}_{3}$. Let us first observe that in the proof of (2.9), assumption $\mathbf{H}_{\mathbf{1}}$, is used in several places in a crucial way. It is also well known that without the boundedness of increments, Harnack's inequalities are no longer satisfied $[1,26]$. On the other hand our main estimate (2.15) cannot hold for an arbitrary $\pi: \mathbb{Z}^{d} \times \mathbb{Z}^{d} \rightarrow[0,1]$. For (2.15) to hold, $\pi$ itself has to have a Gaussian decay at infinity. Decay conditions ensuring the validity of elliptic Harnack inequalities for random walks with infinite range are given in $[1,26]$. An extension to spatially inhomogeneous walks is far from obvious because there are significant differences between the reversible random walks considered in $[1,26]$ and the spatially inhomogeneous walks.

Secondly, the symmetry assumption $\mathbf{H}_{2}$ can be replaced by a centering assumption in order to derive (2.9) (see [22]), but for the analogue of (2.9) to be satisfied by the adjoint walk introduced in $\$ 2.2$, we need symmetry (see [30]). It should be however emphasized that the symmetry assumption was imposed in [30] in order to ease technical difficulties and that there should be no conceptual obstacle to extend the result in this context. It is even likely that our main estimate extends to non-homogeneous random walks with asymptotically zero drifts, like the one considered in [27, 28].

Concerning the ellipticity assumption $\mathbf{H}_{3}$, this assumption was introduced by Lawler in [25] and is often assumed in the random walk in random environment literature, see e.g. [36] . It is plausible that it can be replaced by a weaker spatial non-degeneracy condition, or even an $n_{0}$-step regularity condition of the type [27, Assumption (A1)]. It would be interesting to show that our main estimate (2.15) remains true under a much weaker hypothesis than $\mathbf{H}_{3}$, provided that a lower control on how the transition probabilities $\pi(x, e)$ approach 0 is imposed.

\section{Proof of Theorem 2.12}

The proof consists of successive applications of Harnack principles to well chosen $L$ - and $\tilde{L}$-caloric functions related to the kernels $p_{n}(x, y)$ and $\tilde{p}_{n}(x, y)$. Depending on the position of $x$ and $y$ relative to the boundary, we use the parabolic Harnack principle and/or the boundary Harnack principle to perform various comparisons involving the functions $u$ and $\tilde{u}$. These comparisons lead to upper estimates of the kernel $p_{n}(x, y)$ by the main factors that appear in the right hand side of (2.15). A technical lemma whose proof is postponed to the end of this section is used to incorporate the additional Gaussian factor.

Lemma 3.1. Let the notation be as above. Fix $x, y \in \mathcal{O}_{+}^{d}$ and $n=1,2, \ldots$, such that $\operatorname{dist}\left(x, \partial \mathcal{O}_{+}^{d}\right) \leq c_{1} \sqrt{n}$ and $|x-y| \geq c_{1} \sqrt{n}$, for some $c_{1}>0$. Let $c>0$. Then, there exists $C>0$ depending only on $d, \alpha, \Gamma, c_{1}$ and $c$ such that:

$$
\sum_{z \in \mathcal{O}_{+}^{d},|z-x| \geq c|x-y|} p_{n}(x, z) \leq \frac{C u(x)}{u\left(x_{\sqrt{n}}\right)} \exp \left(-\frac{|x-y|^{2}}{C n}\right)
$$




$$
\text { and } \sum_{z \in \mathcal{O}_{+}^{d},|z-x| \geq c|x-y|} \tilde{p}_{n}(x, z) \leq \frac{C \tilde{u}(x)}{\tilde{u}\left(x_{\sqrt{n}}\right)} \exp \left(-\frac{|x-y|^{2}}{C n}\right) .
$$

This lemma constitutes both a refinement of estimate [31, (4.14)] and a generalization of [2, Theorem 2.2]. It is its statement in this general form which allows to improve the proofs of [31] elaborated in the context of the half-space. Its analogue for the half-space allows to obtain the improvement (2.17).

Proof of Theorem 2.12. We will distinguish two cases depending on whether the starting state $x$ is close or far from the boundary: $\operatorname{dist}\left(x, \partial \mathcal{O}_{+}^{d}\right) \leq c_{0} \sqrt{n}$ or $\operatorname{dist}\left(x, \partial \mathcal{O}_{+}^{d}\right) \geq$ $c_{0} \sqrt{n}$, where $c_{0}$ is a fixed constant verifying $c_{0}<1 / 6 K_{0}, K_{0}$ being the larger of the two constants appearing in theorems 2.6 and 2.10 .

3.1 Case 1: the starting state $x$ is close to the boundary: $\operatorname{dist}\left(x, \partial \mathcal{O}_{+}^{d}\right) \leq c_{0} \sqrt{n}$.

Introduce $\bar{x} \in \partial \mathcal{O}_{+}^{d}$ satisfying $\operatorname{dist}\left(x, \partial \mathcal{O}_{+}^{d}\right)=|x-\bar{x}| \leq c_{0} \sqrt{n}$. We employ the boundary Harnack principle (Theorem 2.6 with a particular choice of the parameters, namely $r=r_{n}=c_{0} \sqrt{n}, s=n$ and $y=\bar{x}$ ) to compare the two $L$-caloric functions $F_{1}(x, k)=$ $p_{k}(x, y), \quad F_{2}(x, k)=u(x), \quad x \in \mathcal{O}_{+}^{d}, \quad k=1,2, \ldots$ (where $y$ is fixed). We then apply several times the parabolic Harnack principle (Theorem 2.5), we obtain

$$
p_{n}(x, y) \leq \frac{C u(x)}{u\left(x_{\sqrt{n}}\right)} \inf _{z \in B_{c \sqrt{n}}\left(x_{\sqrt{n}}\right)} p_{\lfloor C n\rfloor}(z, y) .
$$

Thanks to identity (2.14) involving the adjoint kernel, we deduce

$$
p_{n}(x, y) \leq \frac{C u(x)}{u\left(x_{\sqrt{n}}\right)} \inf _{z \in B_{c \sqrt{n}}\left(x_{\sqrt{n}}\right)} \tilde{p}_{\lfloor C n\rfloor}(y, z) \frac{m(y)}{m(z)} .
$$

Hence, for all $z \in B_{c \sqrt{n}}\left(x_{\sqrt{n}}\right)$, one has $u\left(x_{\sqrt{n}}\right) m(z) p_{n}(x, y) \leq C u(x) m(y) \tilde{p}_{\lfloor C n\rfloor}(y, z)$. Taking the sum over $z \in B_{c \sqrt{n}}\left(x_{\sqrt{n}}\right)$ and using the comparison volume inequality (2.6), one gets

$$
p_{n}(x, y) \leq \frac{C u(x) m(y)}{u\left(x_{\sqrt{n}}\right) v(x, \sqrt{n})} \sum_{z \in B_{c \sqrt{n}}\left(x_{\sqrt{n}}\right)} \tilde{p}_{\lfloor C n\rfloor}(y, z) .
$$

We shall discuss two subcases.

\subsubsection{The states $x$ and $y$ are close to each others: $|x-y| \leq c_{0} \sqrt{n}$.}

Since, we supposed above that $\operatorname{dist}\left(x, \partial \mathcal{O}_{+}^{d}\right) \leq c_{0} \sqrt{n}$, then one can also assume here that $\operatorname{dist}\left(y, \partial \mathcal{O}_{+}^{d}\right) \leq 2 c_{0} \sqrt{n}$. Thus using the same reasoning leading to relation (3.3) applied this time with $\tilde{p}_{n}$ and $\tilde{u}$, we get

$$
\tilde{p}_{n}(y, z) \leq \frac{C \tilde{u}(y)}{\tilde{u}\left(y_{\sqrt{n}}\right)} \tilde{p}_{\lfloor C n\rfloor}\left(y_{\sqrt{n}}, z\right), \text { for all } z \in \mathcal{O}_{+}^{d} .
$$

Therefore, coming back to relation (3.4), using (3.5) and remembering that

$$
\sum_{z \in B_{c \sqrt{n}}\left(x_{\sqrt{n}}\right)} \tilde{p}_{\lfloor C n\rfloor}\left(y_{\sqrt{n}}, z\right)=\mathbb{P}_{y_{\sqrt{n}}}\left[\tilde{S}_{\lfloor C n\rfloor} \in B_{c \sqrt{n}}\left(x_{\sqrt{n}}\right) ; \tilde{\tau}>\lfloor C n\rfloor\right] \leq 1,
$$

we obtain

$$
p_{n}(x, y) \leq \frac{C u(x) m(y) \tilde{u}(y)}{u\left(x_{\sqrt{n}}\right) \tilde{u}\left(y_{\sqrt{n}}\right) v(x, \sqrt{n})} .
$$

Now, having $|x-y| \leq c_{0} \sqrt{n}$ implies $\exp \left(-|x-y|^{2} / C n\right) \geq c$. Estimate (3.6) can then be rewritten as

$$
p_{n}(x, y) \leq \frac{C u(x) \tilde{u}(y) m(y)}{\tilde{u}\left(x_{\sqrt{n}}\right) u\left(y_{\sqrt{n}}\right) v(x, \sqrt{n})} \exp \left(-\frac{|x-y|^{2}}{C n}\right) .
$$


Inhomogeneous random walks on the orthant

3.1.2 The states $x$ and $y$ are away from each others: $|x-y| \geq c_{0} \sqrt{n}$.

In this case, we will also discuss two situations.

Fix $c_{1}>0$.

- First situation. Suppose that $\operatorname{dist}\left(y, \partial \mathcal{O}_{+}^{d}\right) \geq c_{1} \sqrt{n}$. In this configuration, we first apply the adjoint parabolic Harnack principal to the $\tilde{L}$-caloric function $\tilde{F}_{1}$ defined by: $\tilde{F}_{1}(w, k)=\tilde{p}_{k}(w, x), w \in \mathcal{O}_{+}^{d}, k=1,2, \ldots$, where $x$ is fixed. One obtains $\tilde{p}_{n}(y, x) \leq$ $C \inf _{z \in B_{c \sqrt{n}}(y)} \tilde{p}_{\lfloor C n\rfloor}(z, x)$, where the constant $c>0$ is assumed small enough. Then, using relation (2.14), one obtains $p_{n}(x, y) m(z) \leq C p_{\lfloor C n\rfloor}(x, z) m(y)$, for all $z \in B_{c \sqrt{n}}(y)$. Summing over $z \in B_{c \sqrt{n}}(y)$, gives

$$
p_{n}(x, y) \frac{v(y, c \sqrt{n})}{m(y)} \leq C \sum_{z \in B_{c \sqrt{n}}(y)} p_{\lfloor C n\rfloor}(x, z) .
$$

The fact that we have supposed that $|x-y| \geq c_{0} \sqrt{n}$ implies that for $z \in B_{c \sqrt{n}}(y)$, one has

$$
|z-x| \geq c_{2}|x-y|
$$

for some $c_{2}>0$. Combining relations (3.7), (2.6) and (3.8) one obtains

$$
p_{n}(x, y) \leq \frac{C m(y)}{v(x, \sqrt{n})}\left(1+\frac{|x-y|}{\sqrt{n}}\right)^{N} \sum_{z \in \mathcal{O}_{+}^{d},|z-x| \geq c_{2}|x-y|} p_{\lfloor C n\rfloor}(x, z) .
$$

One shall now use (3.1) and conclude that

$$
p_{n}(x, y) \leq \frac{C u(x) m(y)}{u\left(x_{\sqrt{n}}\right) v(x, \sqrt{n})} \exp \left(-\frac{|x-y|^{2}}{C n}\right) .
$$

Moreover, the adjoint parabolic Harnack principle leads to $\tilde{u}(y) \geq c \tilde{u}\left(y_{\sqrt{n}}\right)$. Estimate (2.15) is an immediate consequence of this remark and (3.9).

- Second situation. Assume that $\operatorname{dist}\left(y, \partial \mathcal{O}_{+}^{d}\right) \leq c_{1} \sqrt{n}$. Taking into account that $|x-y| \geq c_{0} \sqrt{n}$, it is clear that if $z \in B_{c \sqrt{n}}\left(x_{\sqrt{n}}\right)$, and $c_{1}>0$ is small enough, then $|z-y| \geq c_{3}|x-y|$, for an appropriate $c_{3}>0$. This together with relation (3.4) imply

$$
p_{n}(x, y) \leq \frac{C u(x) m(y)}{u\left(x_{\sqrt{n}}\right) v(x, \sqrt{n})} \sum_{z \in \mathcal{O}_{+}^{d},|z-y| \geq c_{3}|x-y|} \tilde{p}_{\lfloor C n\rfloor}(y, z) .
$$

Since, we assumed in this case that $\operatorname{dist}\left(y, \partial \mathcal{O}_{+}^{d}\right) \leq c_{1} \sqrt{n}$, according to (3.2), we get

$$
\sum_{z \in \mathcal{O}_{+}^{d},|z-y| \geq c_{3}|x-y|} \tilde{p}_{\lfloor C n\rfloor}(y, z) \leq \frac{C \widetilde{u}(y)}{\widetilde{u}\left(y_{\sqrt{n}}\right)} \exp \left(-\frac{|x-y|^{2}}{C n}\right) .
$$

Estimate (2.15) in this case follows from relations (3.10) and (3.11).

3.2 Case 2: the starting state $x$ is away from the boundary: $\operatorname{dist}\left(x, \partial \mathcal{O}_{+}^{d}\right) \geq c_{0} \sqrt{n}$.

We first observe that if $y$ is away from the boundary: $\operatorname{dist}\left(y, \partial \mathcal{O}_{+}^{d}\right) \geq c_{1} \sqrt{n}$, for some fixed $c_{1}>0$, it suffices then to dominate the kernel $p_{n}(x, y)$ by the global transition kernel $p_{n}^{Z^{d}}(x, y)$. Using (2.7) and applying the Harnack principle, which implies that $u(x) \geq c_{1} u\left(x_{\sqrt{n}}\right)$, and its adjoint version, giving $\tilde{u}(y) \geq c_{1} \tilde{u}\left(y_{\sqrt{n}}\right)$, one immediately deduce the upper estimate (2.15). To handle the remaining case where $\operatorname{dist}\left(y, \partial \mathcal{O}_{+}^{d}\right) \leq c_{1} \sqrt{n}$, it suffices to apply the same reasoning as the case 3.1.2 "first situation" to the adjoint process $\left(\tilde{S}_{n}\right)_{n \in \mathbb{N}}$, the estimate (3.2) having to be used instead of (3.1). This completes the proof of Theorem 2.12. 
Inhomogeneous random walks on the orthant

Proof of Lemma 3.1. Let us first observe that if we assume $n \leq C$ it is easy to verify that (3.1) is satisfied simply by using the range boundedness and ellipticity assumptions. Now fix $n \in \mathbb{N}$ large enough and $x, y \in \mathcal{O}_{+}^{d}$, such that $\operatorname{dist}\left(x, \partial \mathcal{O}_{+}^{d}\right) \leq c_{1} \sqrt{n}$ and $|x-y| \geq c_{1} \sqrt{n}$. Let $m_{0}=n$, define $k \in \mathbb{N}$ such that $2^{k}<m_{0} \leq 2^{k+1}$ and set $m_{1}=2^{k-1}$; one has

$$
\begin{aligned}
\sum_{z \in \mathcal{O}_{+}^{d},|z-x| \geq c|x-y|} p_{m_{0}}(x, z) & =\sum_{\substack{z \in \mathcal{O}_{+}^{d},|z-x| \geq c|x-y|\\
}}\left(\sum_{\xi \in \mathcal{O}_{+}^{d}} p_{m_{1}}(x, \xi) p_{m_{0}-m_{1}}(\xi, z)\right) \\
& =S_{1}+S_{2}
\end{aligned}
$$

where the summation range which defines $S_{1}$ is $\mathcal{S}_{1,0}:=\left\{z \in \mathcal{O}_{+}^{d},|z-x| \geq c|x-y|\right\} \times\{\xi \in$ $\left.\mathcal{O}_{+}^{d},|\xi-x|>c \frac{|x-y|}{2}\right\}$ and the one which defines $S_{2}$ is $\mathcal{S}_{2,0}:=\left\{z \in \mathcal{O}_{+}^{d},|z-x| \geq\right.$ $c|x-y|\} \times\left\{\xi \in \mathcal{O}_{+}^{d},|\xi-x| \leq c \frac{|x-y|}{2}\right\}$. We estimate the first sum $S_{1}$ as follows:

$$
S_{1} \leq \sum_{\xi \in \mathcal{O}_{+}^{d},|\xi-x|>c \frac{|x-y|}{2}} p_{m_{1}}(x, \xi)\left(\sum_{z \in \mathcal{O}_{+}^{d}} p_{m_{0}-m_{1}}(\xi, z)\right) \leq \sum_{\xi \in \mathcal{O}_{+}^{d},|\xi-x|>c \frac{|x-y|}{2}} p_{m_{1}}(x, \xi),
$$

where we use the trivial bound $\sum_{z \in \mathcal{O}_{+}^{d}} p_{m_{0}-m_{1}}(\xi, z)=\mathbb{P}_{\xi}\left[\tau>m_{0}-m_{1}\right] \leq 1$. Regarding $S_{2}$, it can dominated by

$$
S_{2} \leq\left(\sum_{\xi \in \mathcal{O}_{+}^{d}} p_{m_{1}}(x, \xi)\right) \sup _{\xi \in \mathcal{O}_{+}^{d}} \sum_{z \in \mathcal{O}_{+}^{d},|z-\xi| \geq c \frac{|x-y|}{2}} p_{m_{0}-m_{1}}^{\mathbb{Z}^{d}}(\xi, z) .
$$

The boundary Harnack principle (Theorem 2.6) used to compare the two caloric functions $U_{1}(x, n)=\sum_{z \in \mathcal{O}_{+}^{d}} p_{n}(x, z)$, and $U_{2}(x, n)=u(x), x \in \mathcal{O}_{+}^{d}, n=1,2, \ldots$ gives

$$
\sum_{\xi \in \mathcal{O}_{+}^{d}} p_{m_{1}}(x, \xi) \leq \frac{C u(x)}{u\left(x_{\sqrt{m}_{1}}\right)}
$$

Estimate (3.15) is deduced exactly like estimate (3.3) if we assume $\operatorname{dist}\left(x, \partial \mathcal{O}_{+}^{d}\right) \leq c_{0} \sqrt{m}_{1}$, where $c_{0}$ is the constant appearing at the beginning of the proof of Theorem 2.12. Moreover, if $\operatorname{dist}\left(x, \partial \mathcal{O}_{+}^{d}\right) \geq c_{0} \sqrt{m}_{1}$, then by Harnack inequality $u(x) \geq c u\left(x_{\sqrt{m}_{1}}\right)$ and (3.15) results from this remark and the obvious inequality $\sum_{\xi \in \mathcal{O}_{+}^{d}} p_{m_{1}}(x, \xi)=\mathbb{P}_{x}[\tau>$ $\left.m_{1}\right] \leq 1$. On the other hand, the upper Gaussian estimate (2.7) and the doubling volume property (2.6) allow to show by elementary considerations that

$$
\sup _{\xi \in \mathcal{O}_{+}^{d}} \sum_{z \in \mathcal{O}_{+}^{d},|z-\xi|>c \frac{|x-y|}{2}} p_{m_{0}-m_{1}}^{\mathbb{Z}^{d}}(\xi, z) \leq C \exp \left(-\frac{c|x-y|^{2}}{\left(m_{0}-m_{1}\right)}\right) .
$$

Combining (3.12), (3.13), (3.14), (3.15) and (3.16), we obtain

$$
\sum_{z \in \mathcal{O}_{+}^{d},|z-x| \geq c|x-y|} p_{m_{0}}(x, z) \leq \sum_{\xi \in \mathcal{O}_{+}^{d},|\xi-x|>c \frac{|x-y|}{2}} p_{m_{1}}(x, \xi)+\frac{C u(x)}{u\left(x_{\sqrt{m_{1}}}\right)} \exp \left(-\frac{c|x-y|^{2}}{\left(m_{0}-m_{1}\right)}\right) .
$$

The argument can be iterated with $m_{j}=2^{k-j}(1 \leq j \leq k)$, replacing $\mathcal{S}_{1,0}$ by $\mathcal{S}_{1, j}:=\{z \in$ $\left.\mathcal{O}_{+}^{d},|z-x| \geq c \frac{|x-y|}{j+1}\right\} \times\left\{\xi \in \mathcal{O}_{+}^{d},|\xi-x|>c \frac{|x-y|}{j+2}\right\}$ and $\mathcal{S}_{2,0}$ by $\mathcal{S}_{2, j}:=\left\{z \in \mathcal{O}_{+}^{d},|z-x| \geq\right.$ 
$\left.c \frac{|x-y|}{j+1}\right\} \times\left\{\xi \in \mathcal{O}_{+}^{d},|\xi-x| \leq c \frac{|x-y|}{j+2}\right\}$. This gives:

$$
\begin{aligned}
\sum_{z \in \mathcal{O}_{+}^{d},|z-x| \geq c \frac{|x-y|}{2}} p_{m_{1}}(x, z) & \leq \sum_{\xi \in \mathcal{O}_{+}^{d},|\xi-x|>c \frac{|x-y|}{3}} p_{m_{2}}(x, \xi)+\frac{C u(x)}{u\left(x \sqrt{m_{2}}\right)} \exp \left(-\frac{c|x-y|^{2}}{4\left(m_{1}-m_{2}\right)}\right) \\
\ldots & p_{m_{j-1}}(x, z) \leq \sum_{\xi \in \mathcal{O}_{+}^{d},|\xi-x|>c \frac{|x-y|}{j+1}} p_{m_{j}}(x, \xi)+\frac{C u(x)}{u\left(x_{\sqrt{m}_{j}}\right)} \exp \left(-\frac{c|x-y|^{2}}{j^{2}\left(m_{j-1}-m_{j}\right)}\right) \\
\sum_{z \in \mathcal{O}_{+}^{d},|z-x| \geq c \frac{|x-y|}{j}} & \sum_{\sum_{z \in \mathcal{O}_{+}^{d},|z-x| \geq c \frac{|x-y|}{k}} p_{m_{k-1}}(x, z)} \sum_{\xi \in \mathcal{O}_{+}^{d},|\xi-x|>c \frac{|x-y|}{k+1}} p_{m_{k}}(x, \xi)+\frac{C u(x)}{u\left(x_{\sqrt{m}_{k}}\right)} \exp \left(-\frac{c|x-y|^{2}}{k^{2}\left(m_{k-1}-m_{k}\right)}\right) .
\end{aligned}
$$

Summing up all these inequalities and observing that $k \leq \log n / \log 2$ (which implies that $|x-y| /(k+1) \geq c \sqrt{n} / \log n)$ leads to dominate the right hand side of (3.12) by:

$$
\sum_{z \in \mathcal{O}_{+}^{d},|z-x| \geq c \text { 尚 }} p_{1}(x, z)+C \sum_{j=0}^{k-1} \frac{u(x)}{u\left(x_{\sqrt{m_{j+1}}}\right)} \exp \left(-\frac{c|x-y|^{2}}{(j+1)^{2}\left(m_{j}-m_{j+1}\right)}\right) .
$$

Using the fact that the walk $\left(S_{n}\right)_{n \in \mathbb{N}}$ has bounded increments, we deduce that the first sum, is zero since $n$ is supposed to be large. To treat the second sum we first use the Harnack inequality to compare $u\left(x_{\sqrt{m_{j-1}}}\right)$ with $u\left(x_{\sqrt{m}_{j}}\right)$ for $j=1, \ldots, k-1$. This is possible because $\operatorname{dist}\left(x_{\sqrt{m_{j-1}}}, \partial \mathcal{O}_{+}^{d}\right) \geq\left\lfloor\sqrt{m_{j-1}}\right\rfloor$ and $\left|x_{\sqrt{m_{j-1}}}-x_{\sqrt{m_{j}}}\right| \leq \sqrt{m_{j-1}}(1-1 / 2 \sqrt{2})$ as soon as $m_{j}$ is large (when $m_{j}$ is small, use the harmonicity of the function $u$ and hypothesis $\left.\mathbf{H}_{3}\right)$. We can then deduce that $u\left(x_{\sqrt{n}}\right) \leq C u\left(x_{\sqrt{m_{1}}}\right) \leq \cdots \leq C^{j+1} u\left(x_{\sqrt{m_{j+1}}}\right)=$ $2^{c(j+1)} u\left(x_{\sqrt{m_{j+1}}}\right)$, which allows us to replace $1 / u\left(x_{\sqrt{m_{j+1}}}\right)$ by $2^{c(j+1)} / u\left(x_{\sqrt{n}}\right)$ and factor $1 / u\left(x_{\sqrt{n}}\right)$ in front of the sum. We finally obtain,

$$
\begin{aligned}
\sum_{z \in \mathcal{O}_{+}^{d},|z-x| \geq c|x-y|} p_{n}(x, z) & \leq \frac{C u(x)}{u\left(x_{\sqrt{n}}\right)} \sum_{j=0}^{+\infty} 2^{c j} \exp \left(-\frac{2^{j}|x-y|^{2}}{C n(j+1)^{2}}\right) \\
& \leq \frac{C u(x)}{u\left(x_{\sqrt{n}}\right)} \exp \left(-\frac{c|x-y|^{2}}{n}\right) .
\end{aligned}
$$

This completes the proof of (3.1). The proof of (3.2) is obtained in an exactly analogous way.

\section{References}

[1] Bass, R. F. and Kumagai, T.: Symmetric Markov chains on $\mathbb{Z}^{d}$ with unbounded range. Trans. Amer. Math. Soc. 360 (2008), 2041-2075. MR-2366974

[2] Ben Salem, N.; Mustapha, S. and Sifi M.: Survival time of a heterogeneous random walks in a quadrant. C.R. Acad. Sci. Paris. Ser. 352 (2014), 797-801. MR-3262910

[3] Billiard, S. and Tran, V.: A general stochastic model for sporophytic self-incompatibility. J. Math. Biol. 64 (2012), 163-210. MR-2864842

[4] Bostan, A.; Bousquet-Mélou,M.; Kauers, M.; and Melczer, S.: On 3-dimensional lattice walks confined to the positive octant. Ann. Comb. 20 (2016), 661-704. MR-3572381

[5] Bouaziz, A.; Mustapha, S. and Sifi, M.: Discrete harmonic functions on an orthant in $\mathbb{Z}^{d}$. Electron. Commun. Probab. 20 (2015), 1-13. MR-3374302

[6] Bousquet-Mélou, M. and Mishna, M.: Walks with small steps in the quarter plane. Contemp. Math. 520 (2010), 1-40. MR-2681853 
Inhomogeneous random walks on the orthant

[7] Buchacher, M. and Kauers, M.: Inhomogeneous Restricted Lattice Walks. Sém. Lothar. Combin. 82B Art. 75 (2020), 12 pp. MR-4098296

[8] Cohen, J. and Boxma, O.: Boundary value problems in queueing system analysis. NorthHolland Mathematics Studies. 79, North-Holland Publishing Co., Amsterdam (1983). MR0703000

[9] Cont, R. and de Larrard, A.: Price dynamics in a Markovian limit order market. SIAM J. Financial Math. 4 (2013), 1-25. MR-3032934

[10] D'Arco, Ph.; Lacivita, V. and Mustapha, S.: Combinatorics meets potential theory. Electronic Journal of Combinatorics. 23 (2018), 2-28.

[11] Denisov, D. and Wachtel, V.: Random walks in cones. Ann. Probab. 43 (2015), 992-1044. MR-3342657

[12] Dyson, F.: A Brownian-motion model for the eigenvalues of a random matrix. J. Mathematical Phys. 3 (1962), 1191-1198. MR-0148397

[13] Fabes, E. B.; Safonov, M. V. and Yuan, Y.: Behavior near the boundary of positive solutions of second order parabolic equations II. Trans. Amer. Math. Soc. 351 (1999), 4947-4961. MR-1665328

[14] Eichelsbacher P. and König, W.: Ordered random walks. Electron. J. Probab. 13 (2008), 1307-1336. MR-2430709

[15] Fayolle, G.; Iasnogorodski, R. and Malyshev, V.: Random walks in the quarter-plane. Algebraic methods, boundary value problems and applications. Applications of Mathematics (New York), 40. Springer-Verlag, Berlin, (1999). MR-1691900 analytic approach

[16] Fisher, M. E.: Walks, walls, wetting, and melting. J. Stat. Phys. 34 (1984), 667-729. MR0751710

[17] Georgiou, N.; Menshikov, M. V.; Mijatović, A. and Wade, A. R.: Anomalous recurrence properties of many-dimensional zero-drift random walks. Adv. App. Proba. 48 (2016), 99-118. MR-3539299

[18] Gyrya, P. and Saloff-Coste, L.: Neumann and Dirichlet heat kernels in inner uniform domains. Astérisque 336 (2011), SMF. MR-2807275

[19] König, W. and Schmid, P.: Random walks conditioned to stay in Weyl chambers of type C and D. Electron. Commun. Probab. 15 (2010) 286-296. MR-2670195

[20] Krylov, N. V. and Safanov, M. V.: Certains properties of solutions of parabolic equations with mesurable coefficients. Izv. Acad. Nauk. SSSR. 40 (1980), 161-175.

[21] Kuo, H. J. and Trudinger, N. S.: Local estimates for parabolic difference operators. J. Differential Equations 122 (1995), 398-413. MR-1355897

[22] Kuo, H. J. and Trudinger, N. S.: Evolving monotone difference operators on general space-time meshes. Duke Math. J. 91 (1998), 587-607. MR-1604175

[23] Kurkova, I. and Rashel, K.: Random walks in the quarter plane. Bull. Soc. Math. France. 139 (2011), 341-387. MR-2869310

[24] Lafitte-Godillon, P.; Raschel, K. and Tran, V. C.: Extinction probabilities for a distylous plant population modeled by an non-homogeneous random walk on the positive quadrant. SIAM J. Appl. Math. 73 (2013), 700-722. MR-3035492

[25] Lawler, G.F.: Estimates for Differences and Harnack Inequality for Difference Operators Coming from Random Walks with Symmetric, Spatially Inhomogeneous, Increments. Proc. London Math. Soc. 3 (1991), 552-568. MR-1127149

[26] Lawler, G.F. and Polaski, T. W.: Harnack inequalities and difference estimates for random walks with infinite range. J. Theoret. Probab. 6 (1993), 781-802. MR-1245395

[27] MacPhee, I. M.; Menshikov, M. V. and Wade, A.R.: Angular asymptotics for multi-dimensional non-homogeneous random walks with asymptotically zero drift. Markov Process. Related Fields 16 (2010), Issue 2, 351-388. MR-2666858

[28] MacPhee, I. M.; Menshikov, M. V. and Wade, A.R.: Moments of Exit Times from Wedges for Non-homogeneous Random Walks with Asymptotically Zero Drifts. J. Theoret. Probab. 26 (2013), Issue 1, 1-30. MR-3023832 
Inhomogeneous random walks on the orthant

[29] Menshikov, M.V.; Popov, S. and Wade, A. R.: Non-homogenous random walks, Lyapunov function methods for near-critical stochastic systems. Camb. Tracts in Maths. 209, Camb. Univ. Press, (2017). MR-3587911

[30] Mustapha, S.: Gaussian estimates for spatially inhomogeneous random walks on $\mathbb{Z}^{d}$. Ann. Probab. 34 (2006), 264-283. MR-2206348

[31] Mustapha, S.: Gambler's ruin estimates for random walks with symmetric spatially inhomogeneous increments. Bernoulli 13 (2007), 131-147. MR-2307398

[32] Raschel, K.: Random walks in the quarter plane, discrete harmonic functions and conformal mappings, with an appendix by Sandro Franceschi. Stochastic Process. Appli. 124 (2014), 3147-3178. MR-3231615

[33] Stembridge, J.: Nonintersecting paths, Pfaffians, and plane partitions. Adv. Math. 83 (1990), 96-131. MR-1069389

[34] Sturm, K. T.: Analysis on local Dirichlet spaces III. The parabolic Harnack inequality. J. Math. pures 75 (1996), 273-297. MR-1387522

[35] Varopoulos, N. Th.: Potential theory in conical domains. Math. Proc. Cambridge Philos. Soc. 125 (1999), 335-384. MR-1643806

[36] Zeitouni, O.: Random walks in random environment. Lectures on probability theory and statistics, 189-312, Lecture Notes in Math., 1837, Springer, Berlin, (2004). MR-2071631

Acknowledgments. We wish to thank the referees for many useful remarks and comments; their suggestions really improved the presentation of this manuscript. 